\title{
Model Planetaries with Rapidly Evolving Central Stars: A Case for FG Sge
}

\author{
K. Kifonidis, D. Schönberner
}

Astrophysikalisches Institut Potsdam, Potsdam, Germany

Ever since the pioneering work of Schönberner $(1979$, A\&A, 79, 108) and Iben $(1984$, ApJ, 277, 333) who showed that the evolution of post-AGB remnants might be affected by late thermal pulses of the helium-burning shell, resulting in a temporary growth of these objects to red giant dimensions, many attempts were made to explain a number of puzzling objects, among them the well-known variable central star FG Sge as well as the R CrB and PG 1159 stars, by this so-called "born-again AGB" scenario (Iben et al. 1983, ApJ, 264, 605; Iben \& MacDonald 1995, in: White Dwarfs, Springer, p. 48). However, it is still not clear if the frequency of occurrence of such events is high enough as to be consistent with the number of born-again candidates. This is due to the very short evolutionary timescales during the pulse and the character of the post-pulse evolution which resembles the first post-AGB phase and makes it difficult for an observer to distinguish such objects from "normal" central stars.

In such cases the planetary shell expelled earlier might contain valuable information about the evolutionary history of the star. To investigate this question in detail we have followed the radiation gasdynamical evolution of a planetary nebula around the $0.625 \mathrm{M}_{\odot}$ thermally pulsing post-AGB stellar model of Blöcker (1995, A\&A, 299, 755) which mimics closely the observed brightness evolution of FG Sge, i.e. it evolves back to the AGB within about 100 years. We used a newly developed radiation hydrodynamics code (Marten \& Szczerba 1996, A\&A, submitted) with full consideration of all time-dependent processes (ionization, recombination, heating, cooling). This is absolutely essential in order to obtain meaningful results since the evolutionary timescale of the central star becomes comparable to those of recombination and cooling.

In general lines the evolution proceeds analogous to the sperical models of Mellema (1994, A\&A, 290, 915) and Marten (1994, PhD thesis) as long as the star traverses the HRD from low to high temperatures for the first time and its radiation field and wind becomes more energetical: the model evolves into a typical two-shell structure. About 150 years after the onset of the thermal pulse when the star is back to AGB, the nebular shells are recombining, cooling and being compressed due to their low thermal pressure since heating by photoionization is extinguished. When the star heats up again, an R-type ionization front forms, causing only minor dynamical effects due to the meanwhile low densities. Therefore, the formerly compressed outer shell becomes visible as a limb-brightened structure. It should be possible to distinguish such objects from ordinary nebulae by their morphology alone, without resorting to such poorly measurable quantities as radii or ages.

An inspection of the observed surface-brightness profiles of Chu et al. (1987, ApJS, $64,529)$ indicates that such nebulae are indeed very rare since we could not attribute such a structure with certainty to any of the 25 cases published. 